\title{
Imaging compact supermassive binary black holes with Very Long Baseline Interferometry
}

\author{
G. B. Taylor,${ }^{1}$ C. Rodriguez,${ }^{1}$ R. T. Zavala,${ }^{2}$ A. B. Peck,${ }^{3}$ \\ L. K. Pollack ${ }^{4}$ and R. W. Romani ${ }^{5}$ \\ ${ }^{1}$ Department of Physics and Astronomy, Univ. of New Mexico, Albuquerque, NM 87131, USA \\ ${ }^{2}$ United States Naval Observatory, Flagstaff Station, AZ 86001, USA \\ ${ }^{3}$ Harvard-Smithsonian CfA, SMA Project, 645 N. A'ohoku Pl, Hilo, HI 96721, USA \\ ${ }^{4}$ Department of Astronomy \& Astrophysics, University of California at Santa Cruz, \\ Santa Cruz, CA 95064, USA \\ ${ }^{5}$ Department of Physics, Stanford University, Stanford, CA 94305 USA \\ email: gbtaylor@unm.edu
}

\begin{abstract}
We report on the discovery of a supermassive binary black-hole (SBBH) system in the radio galaxy $0402+379$, with a projected separation between the two black holes of just $7.3 \mathrm{pc}$. This is the most compact SBBH pair yet imaged by more than two orders of magnitude. These results are based upon multi-frequency imaging using the Very Long Baseline Array (VLBA) which reveal two compact, variable, flat-spectrum, active nuclei within the elliptical host galaxy of $0402+379$. Multi-epoch observations from the VLBA also provide constraints on the total mass and dynamics of the system. The two nuclei appear stationary while the jets emanating from the weaker of the two nuclei appear to move out and terminate in bright hot spots. The discovery of this system has implications for the number of compact binary black holes that might be sources of gravitational radiation. The VLBI Imaging and Polarimetry Survey (VIPS) currently underway should discover several more SBBHs.
\end{abstract}

Keywords. Black hole physics - gravitational waves - galaxies: active - radio continuum: galaxies

\section{Introduction}

Given that most galaxies harbor supermassive black holes at their centers, and that galaxy mergers are common, binary black holes should likewise be common. Yet very few systems have been found, perhaps because they proceed rapidly to parsec-scale separations which cannot be resolved by current X-ray or optical telescopes. Fortunately, in the case where both black holes are radio loud, they can be imaged using Very Long Baseline Interferometry (VLBI). An understanding of the evolution and formation of these systems is important for an understanding of the evolution and formation of galaxies in general.

Our ability to resolve both supermassive black holes in any given binary system depends on the separation between them, on their distance from Earth, and on the resolving power of the telescope used. It is believed that the longest timescales in the evolution of a supermassive binary black hole system leading up to coalescence is the stage in which the system is closely bound $(\sim 0.1-10 \mathrm{pc})$, meaning that in most of these systems the black hole pair can only be resolved by VLBI observations, which provides resolutions of milliarcseconds and finer. 

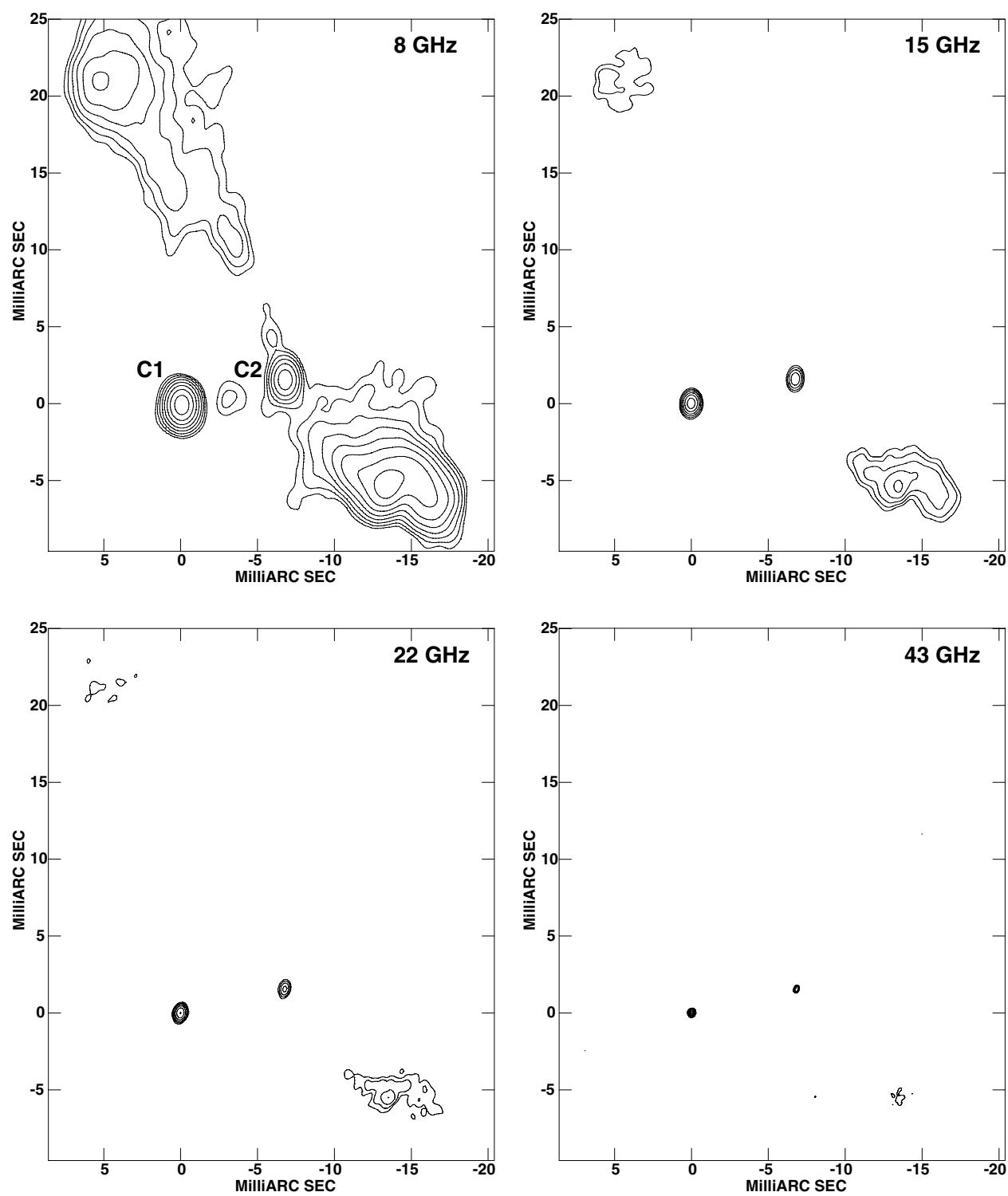

Figure 1. Naturally weighted 2005 VLBA images of $0402+379$ at 8, 15, 22 and $43 \mathrm{GHz}$. Contours are drawn beginning at $3 \sigma$ and increase by factors of 2 thereafter. The labels shown in the 8 $\mathrm{GHz}$ map indicate the positions of the two strong, compact, central components. See Rodriguez et al. (2006) for further details.

Some source properties like X-shaped radio galaxies and double-double radio galaxies, helical radio-jets, double-horned emission line profiles, and semi-periodic variations in lightcurves have been taken as indirect evidence for compact binary black holes though other explanations are possible (see review by Komossa 2003 detailing observational evidence for supermassive black holes binaries). Some wider systems have, however, been found more directly. The ultra luminous galaxy NGC 6240, discovered by the Chandra X-ray observatory, was found to have a pair of active supermassive black holes at its center (Komossa et al. 2003), separated by a distance of $1.4 \mathrm{kpc}$. Another system that has been known for some time is the double AGN (7 kpc separation) constituting the 
radio source $3 \mathrm{C} 75$, which was discovered by the VLA to have two pairs of radio jets (Owen et al. 1985).

Recently, the radio galaxy $0402+379$ was found to contain two central, compact, flat spectrum, variable components (designated C1 and C2 - see Fig. 1), a feature which has not been observed in any other compact source. Multi-frequency VLBA observations are described in greater detail in a recent paper by Rodriguez et al. (2006).

At the redshift of $0402+379$ of 0.055 , for $\mathrm{H}_{0}=75 \mathrm{~km} \mathrm{~s}^{-1} \mathrm{Mpc}^{-1}$, and $\mathrm{q}_{0}=0.5$, gives a scale of 1 mas $=1.06 \mathrm{pc}$.

\section{Constraints on the Orbital Parameters}

A total mass of a few $10^{8} M_{\odot}$ was estimated for the SBBH system in $0402+379$ using VLBA HI observations (Maness et al. 2004) and HET spectroscopy of $\mathrm{H} \alpha$ (Rodriguez et al. 2006). The HET spectrum shows a red shoulder with a velocity of $300 \mathrm{~km} \mathrm{~s}$. At the observed separation of $7.3 \mathrm{pc}$ the implied mass for the system is $1.5 \times 10^{8}(\mathrm{v} / 300$ $\left.\mathrm{km} \mathrm{s}^{-1}\right)^{2}(r / 7.3 \mathrm{pc}) \mathrm{M}_{\odot}$. Using the HI velocity separation of $1000 \mathrm{~km} \mathrm{~s}^{-1}$ on scales of $20 \mathrm{pc}$ gives a larger mass of $5 \times 10^{9} \mathrm{M}_{\odot}$, but the two velocity systems might be probing line-of-sight velocities in the products of a recent merger, and not the orbital motions of the two black holes. Higher resolution observations using a Global VLBI array are planned.

Using a system mass estimate equal to $1.5 \times 10^{8} \mathrm{M}_{\odot}$ and the projected radial separation between them derived from the VLBA images $(7.3 \mathrm{pc})$, we find from Kepler's Laws that the period of rotation for such a binary supermassive black hole system should be $\sim 1.5 \times 10^{5} \mathrm{y}$. This period corresponds to a relative projected velocity between components $\mathrm{C} 1$ and $\mathrm{C} 2$ of $\sim 0.001 \mathrm{c}$. The upper limit found for the relative motion between components $\mathrm{C} 1$ and $\mathrm{C} 2,<0.088 \mathrm{c}$, is consistent with the expected relative velocity assuming a stable, Keplerian orbit. To actually constrain the masses of the black holes would require observations over a longer time baseline $(\sim 100 \mathrm{y})$.

The natural gravitational wave frequency (Hughes 2003) for this system is approximately $2 \times 10^{-13} \mathrm{~Hz}$. This is well below the expected minimum frequency of LISA. Although 0402+379 may be a long way from generating a detectable gravitational wave signal it may represent a source of noise important for future observations of cosmologically produced gravitational radiation. Ultra low frequency gravitational radiation generated during inflation (Hughes 2003) has an upper limit of $10^{-13} \mathrm{~Hz}$. Thus, a population of black hole binaries like 0402+379 may generate substantial noise which could interfere with the detection of the physics of inflation. The current merger time assuming only orbital decay due to gravitational radiation is $\sim 10^{18} \mathrm{y}$. Some other loss of angular momentum (e.g., dynamical friction) will be necessary if this system is to merge in less than a Hubble time.

\section{Statistics of SBBH Systems}

We have identified one SBBH system out of the entire Caltech-Jodrell Bank Flatspectrum survey (CJF) of 293 AGN. Our current incidence is thus $1 / 293$ or $0.34 \%$ with very large uncertainty. Assuming that $10 \%$ of luminous elliptical galaxies host an AGN, and that every galaxy undergoes a merger, then the duration of the SBBH phase should be $\sim 3 \%$ of the lifetime of the elliptical host galaxy or $3 \times 10^{8}$ years. This seems rather long though it is possible that the merger has stalled. Merritt (2006) finds typical stalling radii for Virgo galaxies with comparable black hole masses to those in $0402+379$ of $\sim 7 \mathrm{pc}$ assuming black hole mass ratios of $2: 1$. 
It is not clear whether, at present, dynamical friction losses or gas dissipative effects (see Komossa 2003, Merritt \& Milosavljević 2005) are strong enough to bring the binary to the gravitational radiation loss regime within a Hubble time. This relates to the issue of the probability of catching the binary at its present (modest) separation. Are we seeing a recent merger in the act of core coalescence or has the binary stalled and must now await loss-cone replenishment and/or re-supply of nuclear gas? The fact that both nuclei are active (radio bright) suggests on-going accretion and implies dissipation today. Only a larger sample of imaged active nuclei can address the fraction of the population in this state. The VLBA Imaging and Polarization Survey (VIPS, Taylor et al. 2005) will image 1127 sources. So far we have identified twenty candidate SBBH systems, and observing time on the VLBA has been approved for all of them.

\section{Conclusions}

Based on the compactness, motion, variability, and spectra of the two central components in $0402+379$ we conclude that they are both active nuclei of a single galaxy. This pair of AGN forms the closest binary black hole system yet discovered with a projected separation of $7.3 \mathrm{pc}$. The total mass of the system is estimated to be $1.5 \times 10^{8} \mathrm{M}_{\odot}$, and the gravitational radiation frequency to be $2 \times 10^{-13} \mathrm{~Hz}$. Energy losses due to gravitational radiation are not yet significant, so that other mechanisms must be invoked if the orbit is to decay. $0402+379$ may be the tip of an iceberg for a population of supermassive black hole binaries with parsec scale separations. Such a population may collectively produce significant gravitational wave radiation which may need to be considered for the detection of gravitational radiation in the ultra to very low frequency bands.

\section{Acknowledgements}

The VLBA is operated by the the National Radio Astronomy Observatory, a facility of the National Science Foundation operated under a cooperative agreement by Associated Universities, Inc.

\section{References}

Hughes, S. A. 2003, Annals of Physics, 303, 142

Komossa, S. 2003, AIP Conf. Proc. 686: The Astrophysics of Gravitational Wave Sources, 686, 161

Komossa, S., Burwitz, V., Hasinger, G., Predehl, P., Kaastra, J. S. \& Ikebe, Y. 2003, ApJL, $582, \mathrm{~L} 15$

Maness, H. L., Taylor, G. B., Zavala, R. T., Peck, A. B. \& Pollack, L. K. 2004, ApJ, 602, 123

Merritt, D. 2006, submitted, astro-ph/0603439

Merritt, D., Milosavljević, M. 2005, Living Reviews in Relativity, 8, 8

Owen, F. N., Odea, C. P., Inoue, M. \& Eilek, J. A. 1985, ApJL, 294, L85

Rodriguez, C., Taylor, G. B., Zavala, R. T., Peck, A. B., Pollack, L. K. \& Romani, R. W. 2006, ApJ, 646, 49

Taylor, G. B. et al. 2005, ApJS, 159, 27

XIAn Chen: Why are the binary systems discovered by VLBI all compact systems?

Gregory TAYlor: Actually, some of them are extended. Future low-frequency observations may reveal more extended structures. 